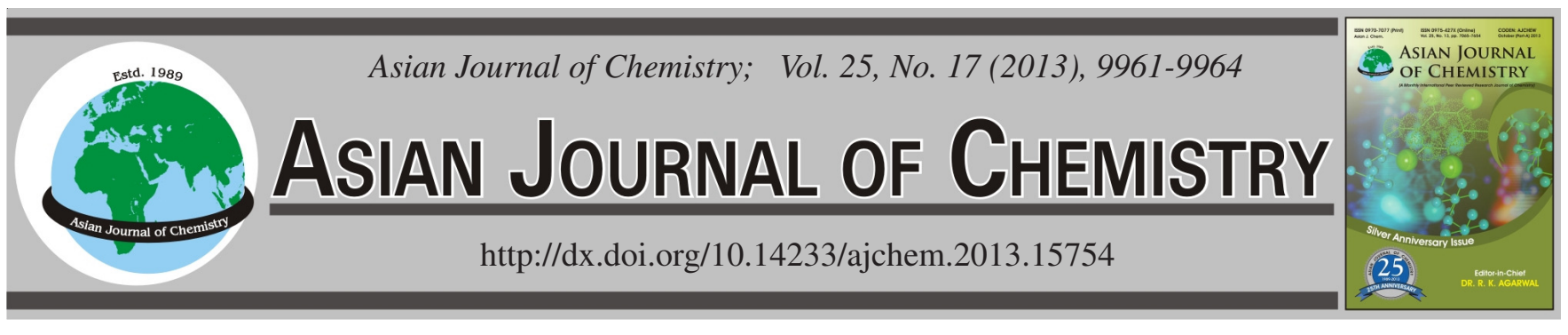

\title{
Antiquorum Sensing and Antibacterial Activity of Silver Nanoparticles Synthesized by Mutant Klebsiella pneumoniae MTCC 3354
}

Mani Arunkumar*, Narayanan Mahesh, Srinivasan Balakumar, Ramalingam Sivakumar and S. Priyadharshni

Department of Chemistry \& Biosciences, Srinivasa Ramanujan Centre, SASTRA University, Kumbakonam-612 001, India

*Corresponding author: Fax: +91 435 2402460; Tel: +91 435 2426823; E-mail: microarun2008@yahoo.in; arunkumar@ src.sastra.edu

Currently there is an urgent need to develop a new strategy for the treatment of antibiotic resistance bacterial pathogen and to reduce the virulent quorum-sensing ability of a microorganism. Microbial synthesis of silver nanoparticles was carried out by using the mutant Klebsiella pneumoniae MTCC 3354 and screened out for antiquorum sensing and antibacterial activity against selected human pathogens. UV-visible spectroscopy and scanning electron microscope study reveals the formation of silver nanoparticles which was predominately spherical shape in the range of 2-20 nm and polydispersed. Silver nanoparticles exhibit enhanced antiquorum sensing and antibacterial activity on selected pathogens. Furthermore, antagonistic activity of amoxicillin, methicillin and ampicillin were increased in the combination of silver nanoparticles against the selected human pathogenic strains. The silver nanoparticles would be alternative strategies for treating the bacterial infection caused by multi-drugs resistance bacteria in upcoming era.

Key Words: Antibacterial activity, Silver nanoparticles, Antiquorum sensing, SEM, Klebsiella pneumoniae.

\section{INTRODUCTION}

Generally, development of green technology for hyper production of metal nanoparticles is an important concept of modern research in the field of nanotechnology due to their unique particle size and shape, which have a wide range of applications in medicals and technological aspects ${ }^{1-4}$. Recently, production of silver nanoparticles can be achieved through many techniques, among the entire one the biological synthesis of silver nanoparticles is gaining importance as it is reliable and ecofriendly ${ }^{5}$. Developments of new strategies for the hyper production of metallic nanoparticles are being considered as an important process in the field of nanotechnolog $\mathrm{y}^{6-8}$. Green synthesis of inorganic nanoparticles by biological methods makes nanoparticles more compatible and environmentally benign'. Recently, among all the biological methods silver nanoparticles (AgNPs) have been extensively synthesized using various bacteria ${ }^{10,11}$, fungi $i^{12,13}$ and plants ${ }^{14,15}$. The biological methods of AgNPs synthesis using microorganism is an ecofriendly, cost effective and alternative method when compared to traditional synthetic methods. Currently, several microorganisms can able to synthesize AgNPs either intra or extracellularly, among the entire microorganism bacteria are considered as a most potent ecofriendly nanofactories ${ }^{16,17}$. In recent years, most of the bacterial and fungal pathogens are found to be resistance to commercially available antimicrobial agents and thereby it causes a serious problem in a human health $^{18,19}$. Nowadays, the opportunity pathogenic bacteria such as Pseudomonas aeruginosa have been able to evolve to become antibiotic resistant which rely on the quorum sensing molecules. Quorum sensing is a cell communication mechanism through which signal molecules called autoinducers activate specific receptors associated with transcription signals for controlling various biochemical processes. Some of these processes are biofilm formation, expression of virulence factors, luminescence, pigment production and mechanisms of resistance to stress conditions $\mathrm{s}^{20}$, which are of major importance in bacterial pathogenesis ${ }^{21,22}$. Furthermore, in most of the bacteria the quorum sensing signal molecules are important for the establishment of infection and can also serve as a switch to pathogenic state. Violacein assay was performed by using bioreporter such as mutant Chromobacterium violaceum CV026, Which lack the synthesis of autoinducer hexanoyl homoserine lactone (C6-HSL) and therefore it requires exogenous addition of $\mathrm{N}$-acylhomoserine lactone autoinducers (AHLs) to undergo quorum sensing and produces the violacein pigment ${ }^{23,24}$. There is an urgent need for search of new antibiotics to treat life-threatening infections caused by multidrug resistance bacterial pathogens. Keeping this in a view, the current aim of our study based on two strategies; to develop a potent mutant strain for hyper-production of AgNPs and also focused to evaluate the antiquorum sensing and antibacterial 
activity of silver nanoparticles synthesized from the mutant Klebsiella pneumoniae.

\section{EXPERIMENTAL}

Muller-Hinton agar media was obtained from Hi-Media, India. Silver nitrate (purity $>99 \%$ ) was used as a precursor in the preparation of silver nanoparticles and AHL (Acyl Homoserine Lactone) were obtained from Sigma. All the glassware's were washed twice with deionized water and stored in air-tight container until use. K. pneumoniae MTCC 3354 and the bacterial test strain such as $S$. aureus MTCC 7443, E. coli MTCC 739 and P. aeruginosa MTCC 2297 were obtained from Microbial Type Culture Collection, Institute of Microbial Technology (IMTECH) and Chandigarh, India.

Random mutagenesis and mutant selection: In the present investigation, two steps of mutagenesis were followed for the development of a highly mutated and stable bacterial strain. The first step of mutagenesis was carried out by exposing the wild strain $K$. pneumoniae MTCC 3354 to various mutagens like UV and ethidium bromide. The mutated strains possessing higher rate of extracellular metabolite for biosynthesis of silver nanoparticles was screened out. During the second step, all the best mutants of first step mutation were again exposed to the same mutagens and double mutated strains were obtained. These strains further screened for the hyper production potential of extracellular metabolite for biosynthesis of silver nanoparticles and among these, best one was isolated and further work was continued on that mutant.

Synthesis of silver nanoparticles by Mutant Klebsiella pneumoniae MTCC 3354: The mutant $K$. pneumoniae MTCC 3354 were grown aerobically in nutrient broth amended with $1 \%$ glucose for the biosynthesis studies. The mutant strain were cultured and incubated at $37^{\circ} \mathrm{C}$, with continuous agitation at $90 \mathrm{rpm}$ for $24 \mathrm{~h}$. After the period of incubation, the cell free filtrate was obtained from the centrifugation $(12,000 \mathrm{rpm}$ for $20 \mathrm{~min}$ ) and filtration process (Whatman filter paper No. 1) and used for further experiments. Hyper production of Silver nanoparticles was performed in $500 \mathrm{~mL}$ Erlenmeyer flasks containing $100 \mathrm{~mL}$ of clean cell free filtrate was bought in contact with $\mathrm{AgNO}_{3}(1 \mathrm{mM})$ and agitated at $28^{\circ} \mathrm{C}$ under $\mathrm{pH}-7$ in dark.

Characterization of AgNPs by using UV-spectroscopy: The preliminary detection of silver nanoparticles produced by the mutant strain was analyzed by visual observation of colour change from colourless to dark brown. The reduced silver ions solution was monitored and confirmed by using a UV-visible spectrophotometer (PerkinElmer's LAMBDA 45) at multiple time intervals from $0-24 \mathrm{~h}$. The absorption spectrum was calculated by using a UV-VIS spectrophotometer in 200 and $700 \mathrm{~nm}$ ranges.

Scanning electron microscopy (SEM): The topological properties of reduced silver nanocolloids were measured by SEM (Hitachi S3000H) at Central Electro Chemical Research Institute (CECRI), Karaikudi and Tamilnadu. The filtrate embedded with silver nanoparticles was subjected to freeze drying under high vacuum tube. The purified freeze dried silver nanocolloids samples were subjected to SEM analysis to reveal the structural pattern, composition and average size of particles. Atleast, two images of the sample with different resolution and magnification were recorded to have a clear representation of its size and shape.

In vitro antibacterial activity assay: In vitro antibacterial activities of silver nanoparticles were investigated against the test pathogenic microorganisms such as $S$. aureus, E. coli and $P$. aeruginosa using well- diffusion method. Using sterile corkborer $(4.5 \mathrm{~mm})$ wells are made on Muller Hinton Agar plates. The selected bacterial pathogenic suspension $(100 \mu \mathrm{L}$ of $10^{4}-10^{5} \mathrm{CFU}$ ) was applied uniformly on the surface of Muller Hinton Agar plates before adding a nanocolloids suspension to the well. Using sterile micropipette, $50 \mu \mathrm{L}(5 \mathrm{mg} / \mathrm{mL})$ of the sample of nanoparticles solution was loaded along with positive control ( $5 \mathrm{mg} / \mathrm{mL}$ ciprofloxacin). After $24 \mathrm{~h}$ of incubation, the zones of inhibition were calculated using high antibiotic zone scale.

Synergistic effect of silver nanoparticles: A disc diffusion method was widely employed to screen the synergistic effects of silver nanocolloids with the combinations of commonly used antibiotic (Amoxicillin, Methicillin and Ampicillin) for the antagonist activity against test bacterial strains. To examine the synergistic effects, each standard antibiotic disc was loaded with $10 \mu \mathrm{L}$ of the freshly prepared AgNPs at a final content of $10 \mu \mathrm{g} /$ disc. Muller-Hinton Agar plates were inoculated with pure cultures of test strain such as $S$. aureus, E. coli and $P$. aeruginosa. Similar experiments were carried out with AgNPs alone. After incubation at $37^{\circ} \mathrm{C}$ for $18 \mathrm{~h}$ the different zones of inhibition were measured.

Antiquorum sensing activity of silver nanoparticles by disc diffusion assay: Antiquorum sensing activity of silver nanoparticles produced by mutant $K$. pneumoniae was assayed by disc diffusion method by using $C$. violaceum as a bioreporter. Luria Bertani agar plates were seeded with $0.1 \mathrm{~mL}$ of approximately diluted (C. $\left.2.5 \times 10^{6} \mathrm{CFU} \mathrm{mL}^{-1}\right)$ freshly grown cultures along with AHLs as exogenous source of quorum sensing molecules. Sterile discs (6 mm diameter) impregnated with different amounts $(5,10,15 \mu \mathrm{L})$ of silver nanocolloids solutions. Solvent and sterile LB broth was used as control. These discs were placed on agar plates overlaid with the indicator strain. Plates were incubated for $18-24 \mathrm{~h}$ at $28^{\circ} \mathrm{C}$ to check the inhibition of pigment violacein production around the well.

\section{RESULTS AND DISCUSSION}

The stable and double mutated strain of K. pneumoniae was screened out by using various mutagenic agents for hyper production of AgNPs. The intense colour change from colourless to yellowish brown was observed in mutant strain rapidly ( $0-3 \mathrm{~h})$ when compared to wild strain, which suggests that the synthesis of silver nanoparticles by the mutant strain is greater than that produced by the wild strain of K. pneumoniae (Fig. 1). The formation of yellowish brown colour of silver nanoparticles is due to the activity of surface plasmon vibrations, which is a characteristic feature of silver nanoparticles ${ }^{25}$.

Similarly, the formation of silver nanoparticles was also confirmed by UV-visible spectrophotometer. The UV-visible spectra showed a strong plasma resonance which was centered approximately at $424 \mathrm{~nm}$ of AgNPs, produced by the mutant strain. It was observed that the maximum absorption occurs at 


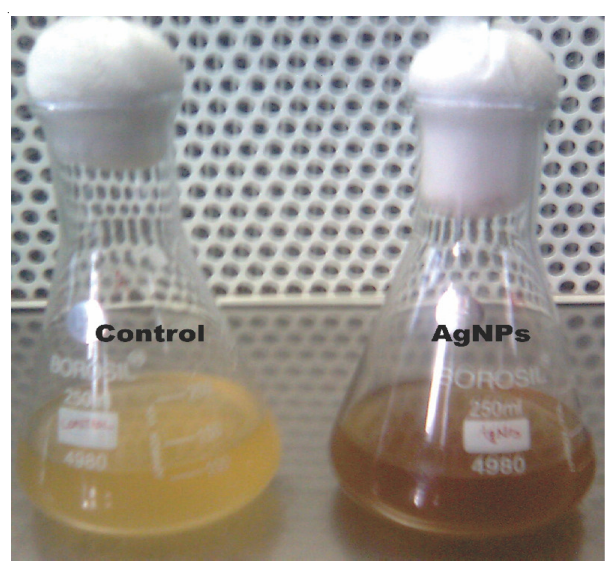

Fig. 1. Synthesis of AgNPs by the mutant strain of K. pneumoniae

$424 \mathrm{~nm}$ (Fig. 2), broad peak represents poly-dispersion of particles. Furthermore, free electrons of AgNPs which give rise to surface plasmon resonance (SPR) absorption band, occurring due to the collective oscillation of electrons of AgNPs in resonance with light wave ${ }^{26}$. The actual mechanism for the synthesis of silver nanocolloids was not yet clearly proved but an enzyme NADH-dependent nitrate reductase is found to be vital enzyme involved in the process ${ }^{27}$.
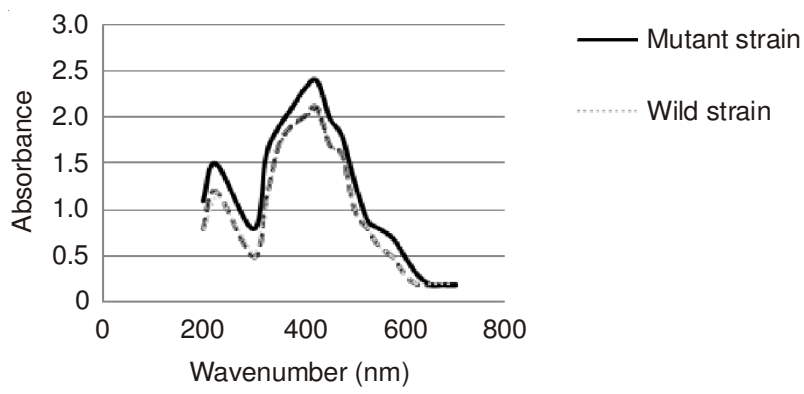

Fig. 2. Absorption spectra (UV-visible spectra) of silver nanoparticles synthesized by wild and Mutant strain of $K$. pneumoniae

SEM analysis reveals the size and structural patterns of silver nanocolloids. Moreover, SEM micrographs of nanoparticles obtained in the filtrate showed that the diameter of the nanoparticles in the solution was above $2-20 \mathrm{~nm}$. The silver nanoparticles are spherical in shape and well distributed without aggregations (Figs. 3 and 4).

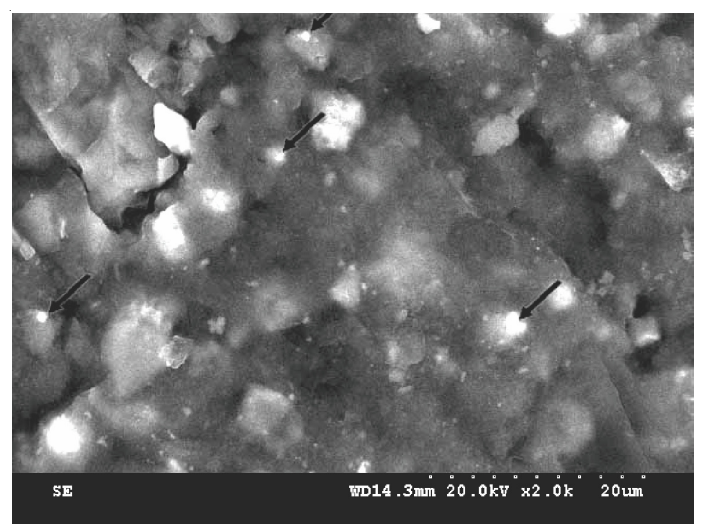

Fig. 3. SEM micrographs of silver nanoparticles obtained by the reaction of $1 \mathrm{mM}$ aqueous silver nitrate solution with mutant strain of Klebsiella pneumoniae (after drying) with $\mathrm{pH}$ 6.0. (Magnification $\times 2,000$ )

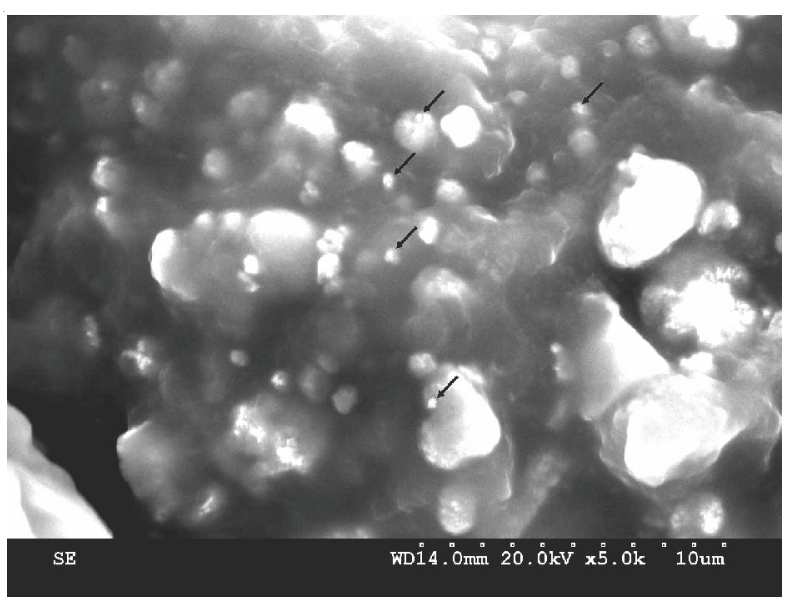

Fig. 4. SEM micrographs of silver nanoparticles obtained by the reaction of $1 \mathrm{mM}$ aqueous silver nitrate solution with mutant strain of Klebsiella pneumoniae (after drying) with $\mathrm{pH}$ 6.0. (Magnification $\times 5,000$ )

In vitro antibacterial activity of AgNPs was examined against three selected pathogenic bacteria, using ciprofloxacin as positive control, an antibacterial agent which is widely employed against many bacterial infections. Fig. 5 represents the zone of inhibition for $50 \mu \mathrm{L}$ of AgNPs solution against test pathogens such as $S$. aureus $(28 \mathrm{~mm})$, E. coli $(32 \mathrm{~mm})$ and $P$. aeruginosa $(35 \mathrm{~mm})$. The antagonistic activity of silver nanoparticles is based on the size, structural pattern of silver nanocolloids, nature of the microbial metabolite and concentration dependent ${ }^{28}$. This study clearly demonstrates that the zone of clearance increased in concentration dependent manner of silver nanoparticles against test pathogenic bacteria. The actual impact of silver nanocolloids as a bactericidal agent against bacteria is not yet clearly established ${ }^{29}$. Furthermore, AgNPs able to pass through the cell membrane of bacteria and thereby it may interact with DNA molecules, thus it arrest the replication process which may lead to the cell death. Synergistic effect of silver nanocolloids in combination with different antibiotics such as methicillin, amoxycillin and ampicillin was investigated against three pathogenic bacteria by using disc diffusion method. The zone of inhibition of silver nanocolloids was examined along with different antibiotic discs with and without AgNPs against selected organisms (Table-1) activity of amoxycillin, ampicillin and methicillin increased in presence of nanoparticles against $P$. aeruginosa, E. coli and $S$. aureus. Methicillin was found to possess highest percentage fold increase, followed by amoxycillin and ampicillin. The maximum antibacterial activity for methicillin in combination with silver nanocolloids was observed against $S$. aureus followed by $P$. aeruginosa and $E$. coli with a percentage fold increase of 100, 30 and $57 \%$, respectively. In case of ampicillin combination with silver nanocolloids the maximum activity was observed against $P$. aeruginosa with $40 \%$ fold increase in antibacterial activity and E. coli for $35 \%$ and followed by $S$. aureus with $33 \%$. The use of amoxycillin with silver nanoparticles showed fold increase in $P$. aeruginosa, E. coli and $S$. aureus with fold ranges from 64, 58 and $54 \%$, respectively.

The enhanced synergetic effects of silver nanocolloids are due the bonding reaction between antibiotic and silver nanoparticles. Moreover, the nanoparticles have large surface 
TABLE-1

SYNERGISTIC EFFECT OF AgNPS AGAINST TEST BACTERIAL PATHOGENS

\begin{tabular}{|c|c|c|c|c|c|c|c|c|c|}
\hline \multirow{3}{*}{ Microorganisms } & \multicolumn{9}{|c|}{ Zone of inhibition (mm) } \\
\hline & \multicolumn{3}{|c|}{ Amoxicillin (Am) } & \multicolumn{3}{|c|}{ Methicillin (M) } & \multicolumn{3}{|c|}{ Ampicillin (A) } \\
\hline & Am & $\begin{array}{c}\mathrm{Am}+ \\
\mathrm{AgNps}\end{array}$ & $\begin{array}{l}\text { Fold increases } \\
\text { in }(\%)\end{array}$ & M & $\begin{array}{c}\mathrm{M}+ \\
\mathrm{AgNps}\end{array}$ & $\begin{array}{l}\text { Fold increases } \\
\text { in }(\%)\end{array}$ & A & $\begin{array}{c}\mathrm{A}+ \\
\mathrm{AgNps}\end{array}$ & $\begin{array}{l}\text { Fold increases } \\
\text { in }(\%)\end{array}$ \\
\hline Staphylococcus aureus & 11 & 17 & 54.54 & 0 & 11 & 100 & 15 & 20 & 33.33 \\
\hline Escherichia coli & 12 & 19 & 58.33 & 10 & 13 & 30.0 & 14 & 19 & 35.71 \\
\hline Pseudomonas aeruginosa & 11 & 18 & 63.63 & 14 & 22 & 57.14 & 10 & 14 & 40.00 \\
\hline
\end{tabular}

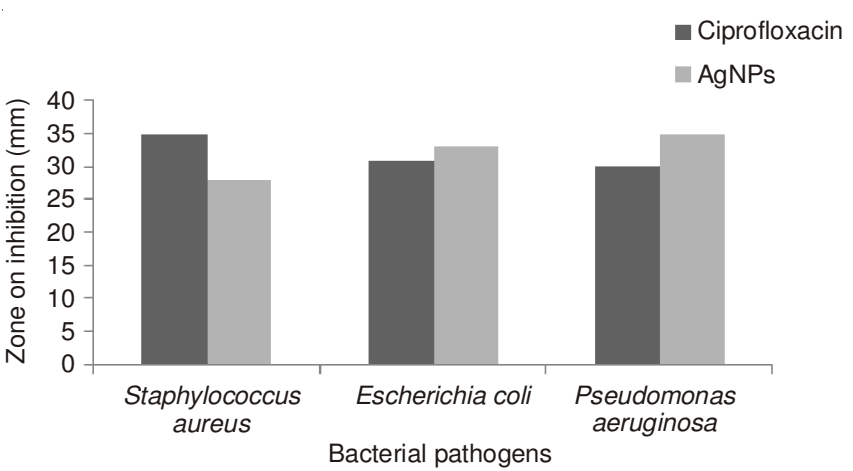

Fig. 5. Antibacterial activity of AgNPs against test bacterial pathogens

area which allows them to closely interact with antibiotics. Most of the antibiotic molecules contain some active groups, which can easily react with AgNPs by chelation ${ }^{30}$. Since the nanoparticles are too small in size, they can come in contact with antibiotics, thereby either it can inhibit peptidoglycan synthesis or AgNPs-antibiotics complex can react with DNA leading to the damage of the bacterial cells ${ }^{31}$.

Chromobacterium violaceum assay was performed with different concentrations $(15,10,5 \mu \mathrm{L})$ of silver nanoparticles synthesized by mutant $K$. pneumoniae by disc diffusion assay using the bioreporter strain CV026. Loss of purple pigment in CV026 cultured with exogenous AHL is indicative of quorum sensing inhibition by the silver nanoparticles synthesized by mutant $K$. pneumoniae. A clear halo zone of inhibition around the wells of varying diameter indicates that quorum sensing inhibition effect was relative to the amount of silver nanocolloids added. Finding in this study, confirms for the first time quorum quenching activity of silver nanoparticles produced by the mutant strain. Furthermore, in the present study the silver nanocolloids exhibit varying degree of antibacterial activity and synergistic effect against selected pathogens.

\section{Conclusion}

We have demonstrated a simple biotechnological process for the hyper-intracellular synthesis of silver nanoparticles using this mutant bacterial strain. Furthermore, this research article aims at reviewing the literature and helping us to understand the ways of communication among bacteria, which further open up the prospects in the treatment of diseases caused by multi drug resistance bacteria.

\section{REFERENCES}

1. R.W. Glomm, J. Disper. Sci. Technol., 26, 389 (2005).

2. W.C. Chan, Biol. Blood Marrow Transplant., 12S, 87 (2006).
3. E. Boisselir and D. Astruc, Chem. Soc. Rev., 38, 1759 (2009).

4. Z. Sadowski, In ed.: D.P. Perez, Biosynthesis and Application of Silver and Gold Nanoparticles, Silver Nanoparticles, ISBN: 978-953-307028-5, InTech, DOI: 10.5772/8508 (2010).

5. S. Rajesh, D. Patric Raja, J.M. Rathi and K. Sahayaraj, J. Biopest., 5S, 119 (2012).

6. S. Ponarulselvam, C. Panneerselvam, K. Murugan, N. Aarthi, K. Kalimuthu and S. Thangamani, Asian Pac. J. Trop. Biomed., 2, 574 (2012).

7. V. Armendariz, J.L. Gardea-Torresday, M. Jose Yacaman, J. Gonzalez, I. Herrera and J.G. Parsons, Gold Nanoparticle Formation by Oat and Wheat Biomasses. Proceedings of Conference on Application of Waste Remediation Technologies to Agricultural Contamination of Water Resources (2002).

8. P. Raveendran, J. Fu and S.L. Wallen, Green Chem., 8, 34 (2006).

9. G. Schmid, Chem. Rev., 92, 1709 (1992).

10. K. Kalimuthu, R.S. Babu, D. Venkataraman, M. Bilal and S. Gurunathan, Colloid Surf. B, 65, 150 (2008).

11. P.R. Chaudhari, S.S. Masurkar, V.B. Shidore and S.P. Kamble, Int. J. Pharm. Biosci., 3, 222 (2012).

12. A. Ahmad, P. Mukherjee, S. Senapathi, D. Mandal, M.I. Khan and R. Kumar, Colloid. Surf. B, 28, 313 (2003).

13. K. Kathiresan, S. Manivannan, M.A. Nabeel and B. Dhivya, Colloids Surf. B, 71, 133 (2009).

14. R. Raut, J.R. Lakkakula, N. Kolakar, V.D. Mendhulkar and S.B. Kashid, Nanomicro Lett., 2, 106 (2010).

15. S.A. Masurkar, P.R. Chaudhari, V.D. Shidore and S.P. Kamble, NanoMicro Lett., 3, 189 (2011).

16. S. Mann, Materials Chemistry, VCH, New York (1996).

17. R. Ragunathan, Digest J. Nanomater. Biostruct., 4, 623 (2009).

18. G.D. Wright, Adv. Drug Deliv Rev., 57, 1451 (2005).

19. M. Gajbhiye, J. Kesharwani, A. Ingle, A. Gade and M. Rai, Nanomed. Nanotechnol. Biol. Med., 5, 382 (2009).

20. J.T.V. Olivero, N.P.C . Pajaro and E. Stashenko, Vitae, 18, 77 (2011).

21. D.A. Vattem, K. Mihalik, S.H. Crixell, R. McLean, Fitoterapia, 78, 302 (2007).

22. F. Bai, Y. Han, J. Chen and X.H. Zhang, Aquaculture, 274, 36 (2008).

23. M. Arunkumar, S.H. Sheikabdulla, R. Sivakumar and N. Mahesh, Global J. Pharmacol., 6, 118 (2012).

24. P.D. Shaw, G. Ping, S.L. Daly, C. Cha, J.E. Cronan, K.L. Rinehart and S.K. Farand, Proc. Nat. Acad. Sci. USA, 94, 6036 (1997).

25. Y.S. Jae and S.K. Beom, Bioprocess Biosyst. Eng., 32, 79 (2009).

26. Y. Roh, R.J. Lauf, A.D. McMillan, C. Zhang, C.J. Rawn and J. Bai, Solid State Commun., 118, 529 (2011).

27. S.S. Nath, D. Chakdar and G. Gope, Nanotrends. Nanotechnol. Appl., 2 (2007).

28. S.K.R. Namasivayam and Avimanyu, Int. J. Pharm. Pharm. Sci., 3, 190 (2011).

29. A. Panacek, L. Kvitek, R. Prucek, M. Kolar, R. Vecerova, N. Pizurova, V. Sharma, T. Nevecna and R. Zboril, J. Phys. Chem. B, 110, 16248 (2006).

30. K.I. Batarseh, J. Antimicrob. Chemother, 54, 546 (2004).

31. S.P. Dhas, A. Mukerjhee and N. Chandrasekaran, Int. J. Pharm. Pharm. Sci., 5, 349 (2013). 